\title{
Adaptabilidade e estabilidade de cultivares de soja em várzea irrigada no Tocantins ${ }^{1}$
}

\author{
Adaptability and stability of soybean cultivars under conditions of varzeas, in \\ Tocantins State Brazil
}

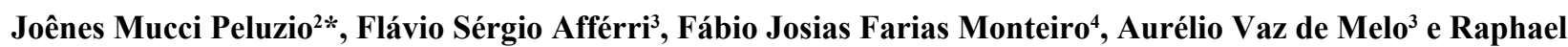 \\ Sanzio Pimenta ${ }^{4}$
}

\begin{abstract}
Resumo - Com o objetivo de avaliar o rendimento de grãos dos cultivares de soja, foram conduzidos três ensaios sob condições de várzea irrigada em Formoso do Araguaia - Tocantins, na entressafra 2007 (15/05; 30/05 e 12/06). O delineamento experimental foi em blocos casualizados com três repetições e dez tratamentos. Os cultivares foram: DM Nobre, A 7002, DM 247, DM 309, DM 339, M-SOY 8411, M-SOY 8914, M-SOY 8866, M-SOY 9350 e FT 106. Na avaliação do desempenho dos cultivares, foi realizado um estudo de adaptabilidade e estabilidade através dos métodos de Eberhart e Russell (1966) e Centróide. O rendimento médio de grãos variou de $1.023 \mathrm{~kg} \mathrm{ha}^{-1}(15 / 05)$ a $1.234 \mathrm{~kg} \mathrm{ha}^{-1}$ (12/06), sendo a média geral de $1.142 \mathrm{~kg} \mathrm{ha}^{-1}$. Baseado na classificação obtida pelas metodologias de Eberhart e Russell (1966) e Centróide, os cultivares DM Nobre, A 7002, M-SOY 9350 e M-SOY 8914 foram mais promissores para cultivo em várzea irrigada. Houve concordância entre as metodologias quanto à classificação dos genótipos.
\end{abstract}

Palavras-chave - Glycine max (L.). Melhoramento-genético. Interação genótipos-ambientes.

\begin{abstract}
In order to evaluate the performance of soybean cultivars, three essays were carried out at Formoso do Araguaia, TO,Brazil, in the inter-cropping 2007 (15/05, 30/05 and 12/06). The experimental design employed was a randomized blocks with ten treatments and tree replications. The treatments were composed by ten soybean cultivar (DM Nobre, NIDERA In 7002, DM 247, DM 309, DM 339, M-SOY 8411, M-SOY 8914, M-SOY 8866, M-SOY 9350 and FT 106). In evaluating the performance of cultivars, a study was conducted of adaptability and stability by the methods of Eberhart and Russell (1966) and centroid. The average grain yield varied from $1023 \mathrm{~kg} \mathrm{ha}^{-1}(15 / 05)$ to $1234 \mathrm{~kg} \mathrm{ha}^{-1}(12 / 06)$, with a general average of $1142 \mathrm{~kg} \mathrm{ha}^{-1}$. Based on the classification obtained by the methods of Eberhart and Russell (1966) and centroid, the cultivars DM Nobre, NIDERA In 7002, M-SOY 9350 and M-SOY 8914, were promising for the cultivation under conditions of intercropping. There was agreement among the methods on the classification of genotypes.
\end{abstract}

Key words - Glycine $\max (\mathrm{L}$.$) . Enhancement-genetic. Genotype-environment.$

\footnotetext{
* Autor para correspondência

${ }^{1}$ Recebido para publicação em 03/08/2009; aprovado em 05/07/2010

Pesquisa desenvolvida junto a Universidade Federal do Tocantins, Campus Universitário de Gurupi

${ }^{2}$ Universidade Federal do Tocantins, Campus Universitário de Palmas, Caixa Postal 66, Palmas-TO, Brasil, 77.410-000, joenesp@mail.uft.edu.br

${ }^{3}$ Universidade Federal do Tocantins, Campus Universitário de Gurupi, Caixa Postal 66, Gurupi-TO, Brasil, 77.402-970, flavio@mail.uft.edu.br, vazdemelo@mail.uft.edu.br

${ }^{4}$ Universidade Federal do Tocantins, Campus Universitário de Palmas, Caixa Postal 66, Palmas-TO, Brasil, 77.410-000, fabio@mail.uft.edu.br, raphael@mail.uft.edu.br
} 


\section{Introdução}

No estado do Tocantins, a soja é a terceira cultura, em termos de participação no valor bruto da produção (CONAB, 2009), sendo cultivada no período de entressafra (maio-junho), em condições de várzea irrigada, sob regime de irrigação subsuperficial (controle do lençol freático), principalmente em Formoso do Araguaia, e no período de safra (novembro-dezembro), em condições de terras altas. Na entressafra, a ausência de chuvas, aliada à baixa umidade relativa do ar e à baixa temperatura noturna, favorecem a obtenção de sementes de boa qualidade. Assim, a produção de soja, nesse período, tem-se tornado altamente atrativa para os produtores, em virtude de o preço da soja, comercializada na forma de sementes, ser compensador. Entretanto, há escasses de estudos sobre o desempenho de cultivares de soja neste período.

Em programas de melhoramento de plantas, a seleção e recomendação de genótipos são precedidas por ensaios em vários ambientes, pois em geral, observa-se que o genótipo mais adequado em um ambiente específico não necessariamente é o mais adequado em outro. A esta variação, dá-se o nome de interação genótipo $\mathrm{x}$ ambiente (ALLARD, 1961; ALLARD; BRADSHAW, 1964; CRUZ et al., 2006; EBERHART; RUSSELL, 1966).

A caracterização da interação genótipo x ambiente, para rendimento de grãos e outras características agronômicas, é um fator importante nos programas de melhoramento, uma vez que dificulta a detecção de diferenças significativas entre os genótipos (ALLARD, 1961; CRUZ et al., 2006; EBERHART; RUSSELL, 1966; FINLAY; WILKINSON, 1963; PELUZIO, 1996; PELUZIO et al., 2008; VERNETTI et al., 1990).

A recomendação de genótipos específicos para cada ambiente (ALLARD; BRADSHAW, 1964), a estratificação de uma área heterogênea em sub-regiões mais homogêneas (TAI, 1971) e a identificação de genótipos fenotipicamente mais estáveis e que respondam previsivelmente às variações ambientais (CRUZ et al., 2006; EBERHART; RUSSELL, 1966; FINLAY; WILKINSON, 1963; VICENTE et al. 2004), têm sido as alternativas propostas para atenuar o efeito da interação genótipos x ambientes. Dentre estas, esta última, representada por estudos de adaptabilidade e estabilidade, é a que tem sido mais amplamente empregada para atenuar o efeito da interação, uma vez que pode ser empregada em diferentes situações (ALLARD, 1961; CRUZ et al., 2006; EBERHART; RUSSELL, 1966; FINLAY; WILKINSON, 1963; PELUZIO, 1996; PELUZIO et al., 2008; VERNETTI et al., 1990).

Segundo Cruz et al., (2006), adaptabilidade é a capacidade de os genótipos aproveitarem vantajosamente o estímulo do ambiente e estabilidade é a capacidade de os genótipos mostrarem um comportamento altamente previsível em função do estímulo do ambiente.

No método proposto por Eberhart e Russell (1966), para cada genótipo é computada uma regressão linear simples da variável dependente, em relação a um índice ambiental (diferença entre a média de cada ambiente e a de todos os ambientes), cujos valores do coeficiente de regressão podem ser positivos ou negativos. Os negativos indicam os ambientes desfavoráveis, evidenciando áreas de cultivo cujo índice tecnológico empregado é baixo ou regiões com condições edáficas e climáticas adversas. Os positivos indicam os ambientes favoráveis, associados às regiões com condições climáticas e edáficas apropriadas à aptidão da cultura ou áreas de cultivo onde se emprega alta tecnologia de produção.

Metodologias baseadas em componentes principais, ou seja, centróide, embora rotineiramente utilizadas emprogramas de melhoramento em estudos de diversidade genética, são pouco utilizadas em estudos da interação genótipo $\mathrm{x}$ ambiente (ROCHA et al., 2005). Nesta metodologia, a variação da performance dos genótipos nos ambientes é dispersa em um plano com poucos eixos, permitindo uma análise simultânea do desempenho de um número elevado de genótipos e, neste sentido, facilitando a interpretação dos resultados. $\mathrm{O}$ conceito de adaptabilidade e estabilidade utilizado no método centróide diferencia dos demais, uma vez que o genótipo de máxima adaptação específica não é aquele que apresenta bom desempenho nos grupo de ambientes favoráveis ou desfavoráveis, mas sim o genótipo que apresenta valores máximos para determinado grupo de ambientes (favoráveis e desfavoráveis) e mínimo para o outro conjunto.

Objetivou-se avaliar o rendimento de grãos e a adaptabilidade e estabilidade de cultivares de soja, em várzea irrigada, no Sul do Estado do Tocantins.

\section{Material e métodos}

Foram realizados três ensaios de competição de cultivares de soja na entressafra 2006 (15/05; 30/05 e 12/06), no município de Formoso do Araguaia, TO (170 m de altitude, $11^{\circ} 45^{\prime}$ S, 4941' W, solo do tipo Gley Pouco Húmico). Cada época de plantio representou um ambiente. $\mathrm{O}$ delineamento experimental utilizado foi o de blocos ao acaso, com quatro repetições e 10 tratamentos. Os tratamentos constaram dos cultivares DM Nobre, DM 247, DM 309, DM 339, A 7002, M-SOY 8411, M-SOY 8914, M-SOY 8866, M-SOY 9350 e FT 106.

A unidade experimental foi composta por quatro fileiras de 5,0 m de comprimento, com espaçamento de $0,40 \mathrm{~m}$ entre fileiras, sendo a área útil da parcela representada pelas duas fileiras centrais, eliminando- 
se $0,50 \mathrm{~m}$ da extremidade de cada fileira. A densidade de semeadura foi realizada com o intuito de se obter 14 plantas por metro linear.

Por ocasião do plantio, em cada ensaio, as sementes foram inoculadas com estirpes de Bradyrhizobium japonicum. Os tratos culturais, como o controle de plantas daninhas, pragas (percevejos e lagartas), doenças (oídio e míldio) e irrigação suplementar por sub-irrigação (elevação do lençol freático), foram realizados sempre que se fizeram necessários.

As plantas, de cada parcela experimental, foram colhidas uma semana após terem apresentado 95\% das vagens maduras. Após a colheita, as plantas foram trilhadas e as sementes pesadas, após estarem secas (12\% de umidade) e limpas, para a determinação do rendimento de grãos.

Foi realizada a análise de variância conjunta dos ensaiose, quandohouvesignificâncianainteraçãocultivares $\mathrm{x}$ ambientes, foi realizada análise de adaptabilidade e estabilidade, segundo os métodos de Eberhart e Russell (1966) e Centróide (ROCHA et al. 2005).

No modelo estatístico, para a análise conjunta, considerou-se fixo o efeito do cultivar e os demais aleatórios.

A metodologia de Eberhart e Russell (1966) leva em consideração, na avaliação dos genótipos, o rendimento médio do genótipo $\left(\mu_{\mathrm{i}}\right)$, o seu coeficiente de regressão $\left(\beta_{1}\right)$ e a variância dos desvios dessa regressão $\left(\sigma^{2}{ }_{\mathrm{di}}\right)$, cujos estimadores são dados por:

$$
\begin{gathered}
\bar{Y}_{i}=\frac{\sum_{j} Y_{i j}}{a} \\
\hat{\beta}_{1}=\frac{\sum_{j} Y_{i j} I_{j}}{\sum_{j} I_{j}^{2}}
\end{gathered}
$$

em que $I_{j}=\frac{\sum_{j} Y_{i j}}{g}-\frac{\sum_{i} \sum_{j} Y_{i j}}{a g}$ (índice ambiental)

$\hat{\sigma}_{\mathrm{di}}^{2}=\frac{\left[\sum_{\mathrm{j}} \mathrm{Y}_{\mathrm{ij}}^{2}-\left(\sum_{\mathrm{j}} \mathrm{Y}_{\mathrm{ij}}\right)^{2} / \mathrm{a}\right]-\left(\sum_{\mathrm{j}} \mathrm{Y}_{\mathrm{ij}} \mathrm{I}_{\mathrm{j}}\right)^{2} / \sum_{\mathrm{j}} \mathrm{I}_{\mathrm{j}}^{2}}{\mathrm{a}-2}$

No método centróide, segundo ROCHA et al. (2005), inicialmente, os ambientes são classificados em favoráveis e desfavoráveis, segundo o índice ambiental proposto por Finlay e Wilkinson (1963).

$\mathrm{I}_{\mathrm{j}}=\frac{1}{\mathrm{~g}} \sum_{\mathrm{i}} \mathrm{Y}_{\mathrm{ij}}-\frac{1}{\mathrm{ag}} \mathrm{Y}$.
Em que: $\mathrm{Y}_{\mathrm{ij}}$ : média do genótipo $\mathrm{i}$, no ambiente $\mathrm{j}$; ${ }^{\mathrm{Y} . .}$ : total das observações; a: número de ambientes; g: número de genótipos.

Após a classificação dos ambientes, são criados quatropontos referenciais(ideótipos), visandorepresentar os genótipos de máxima adaptabilidade geral, máxima adaptabilidade específica a ambientes favoráveis ou desfavoráveis e os genótipos de mínima adaptabilidade. O ideótipo de máxima adaptabilidade geral é aquele que apresenta os valores máximos observados para todos os ambientes estudados (ideótipo I). Os ideótipos de máxima adaptabilidade específica são aqueles que apresentam máxima resposta em ambientes favoráveis e mínima resposta em ambientes desfavoráveis (ideótipo II) ou máxima resposta em ambientes desfavoráveis e mínima em ambientes favoráveis (ideótipo III). O ideótipo de mínima adaptabilidade é aquele que apresenta os menores valores em todos os ambientes estudados (ideótipo IV).

Posteriormente, são comparados os valores de distância cartesiana entre os genótipos e quatro referências ideais (ideótipos). Uma medida de probabilidade espacial pode ser calculada utilizando o inverso da distância entre um tratamento aos quatro ideótipos:

$$
P_{d(i, j)}=\frac{\left[\frac{1}{d i}\right]}{\sum_{i=1}^{4} \frac{1}{d i}}
$$

Em que: $P_{d(i, j)}=$ probabilidade de apresentar padrão de estabilidade semelhante ao j-ésimo centróide; $d i=$ distância do i-ésimo ponto ao j-ésimo centróide.

Os resultados obtidos pelo método centróide também foram comparados com os obtidos por regressão linear, proposta por Eberhart e Russel (1966). Para isso, a similaridades entre os métodos foram comparada quanto ao ordenamento dos genótipos nos ambientes, utilizando a correlação classificatória de Spearman (STEEL; TORRIE, 1980).

\section{Resultados e discussão}

A análise de variância da característica rendimento de grãos (TAB. 1) apresentou efeito significativo das cultivares em todos os ambientes. Os coeficientes de variação foram baixos, indicando boa precisão dos experimentos. Os maiores valores médios de produção foram obtidos no experimento Formoso III, instalado em 12/06/2006. 
Tabela 1 - Rendimento médio de grãos $\left(\bar{Y}_{. j}\right)$, variância dos tratamentos (QMTrat), variância residual (QMR) e coeficiente de variação (CV) de ensaios de competição de cultivares de soja em três ambientes, no Estado do Tocantins, Entressafra 2006

\begin{tabular}{cccccc}
\hline Ambientes $^{\prime 1}$ & $\bar{Y}_{. j}$ & QMTrat & QMR & F & CV (\%) \\
\hline Formoso I & 1023,37 & 78111,51 & 16401,51 & $4,76^{*}$ & 12,5 \\
Formoso II & 1171,16 & 95496,39 & 6656,38 & $14,35^{*}$ & 6,9 \\
Formoso III & 1234,03 & 145970,48 & 4183,23 & $34,89 *$ & 5,2 \\
\hline
\end{tabular}

${ }^{*}=$ significativo a $5 \%$ de probabilidade pelo teste F; ${ }^{1 /}$ Formoso I - Plantio em 15/05/2006; Formoso II - Plantio em 30/05/2006; Formoso III - Plantio em 12/06/2006

A análise de variância conjunta (TAB. 2) mostrou efeitos significativos dos cultivares, ambientes e da interação cultivar $\mathrm{x}$ ambiente. A significância deste último foi do tipo complexa para todos os pares de ambientes (TAB. 3), revela um comportamento diferencial dos cultivares nos ambientes estudados, justificando-se segundo Peluzio et al. (2005), Rocha et al. (2006) e Dias et al. (2009) a determinação da adaptabilidade e estabilidade de comportamento.

Tabela 2 - Resumo da análise de variância conjunta da produtividade de grãos $\left(\mathrm{kg} \mathrm{ha}^{-1}\right)$, de cultivares de soja em três ambientes, no Estado do Tocantins, Entressafra 2006

\begin{tabular}{lccc}
\hline Fonte de variação & GL & Quadrado médio & F \\
\hline Blocos/ambientes & 6 & 2855,7 & 0,314 \\
Ambientes (A) & 2 & 350887,5 & $38,642^{*}$ \\
Cultivares (C) & 9 & 220138,6 & $24,244^{*}$ \\
Interação C x A & 18 & 49719,8 & $5,476^{*}$ \\
Erro médio & 54 & 9080,3 & \\
CV (\%) & 8,3 & & \\
\hline
\end{tabular}

* = significativo a $5 \%$ de probabilidade pelo teste $\mathrm{F}$

Tabela 3 - Pares de ambientes, correlação entre ambientes e porcentagem da parte complexa resultante da decomposição da interação entre genótipos e pares de ambiente, segundo metodologia de Cruz e Castoldi (1991), nos ensaios de competição de cultivares de soja em três ambientes, no Estado do Tocantins, Entressafra 2006

\begin{tabular}{lcc}
\hline \multicolumn{1}{c}{ Pares de ambientes $^{1 /}$} & Correlação & $\begin{array}{c}\text { Parte } \\
\text { complexa da } \\
\text { interação }\end{array}$ \\
\hline Formoso I e Formoso II & 0,260 & 75,76 \\
Formoso I e Formoso III & 0,515 & 64,52 \\
Formoso II e Formoso III & 0,612 & 52,01 \\
\hline
\end{tabular}

${ }^{1 / F o r m o s o ~ I ~-15 / 05 / 2006 ; ~ F o r m o s o ~ I I ~-~ 30 / 05 / 2006 ; ~ F o r m o s o ~ I I I ~-~}$ $12 / 06 / 2006$
O rendimento médio de grãos variou de $1.023 \mathrm{~kg} \mathrm{ha}^{-1}$ (Formoso I - 15/05) a $1.234 \mathrm{~kg} \mathrm{ha}^{-1}$ (Formoso III - 12/06), com média geral entre os ambientes de $1.142 \mathrm{~kg} \mathrm{ha}^{-1}$ (Tabela 4). O cultivar DM Nobre alcançou a maior média geral $(1.338 \mathrm{~kg}$. ha-1) e alcançou um maior rendimento em Formoso I (1.233 $\left.\mathrm{kg} \mathrm{ha}^{-1}\right)$ e Formoso III (1.481 $\left.\mathrm{kg} \mathrm{ha}^{-1}\right)$. O maior rendimento foi obtido por M-SOY 8914 em Formoso II (30/05) ( $\left.1.433 \mathrm{~kg} \mathrm{ha}^{-1}\right)$. O cultivar DM 247 apresentou a menor média geral $(823 \mathrm{~kg}$. ha-1) e teve um maior rendimento em Formoso II (790 kg ha-1) e Formoso III (879 $\left.\mathrm{kg} \mathrm{ha}^{-1}\right)$. O menor rendimento foi obtido por FT-106 em Formoso I (773 kg ha-1).

As estimativas das médias dos genótipos, dos coeficientes de regressão e dos componentes dos desvios da regressão e coeficiente de determinação, de cada cultivar, obtidas pelo método de Eberhart e Russell (1966), encontram-se na Tabela 5.

Pelo método de Eberhart e Russell (1966) foram caracterizados como ambientes favoráveis os ensaios Formoso II (30/05) e Formoso III (12/06). Por outro lado, por propiciar índice ambiental negativo, o ensaio instalado em 15/05 (Formoso I), foi considerado como desfavorável devido, principalmente, à presença de temperaturas mais altas durante a fase vegetativa, resultando em efeitos adversos na taxa de crescimento das plantas e, por conseguinte, no rendimento de grãos.

Os cultivares DM Nobre, NIDERA A 7002, DM 309, M-SOY 9350 e DM 247 apresentaram coeficiente de regressão igual à unidade $\left(\beta_{1 \mathrm{i}}=1\right)$ e desvio da regressão não significativo $\left(\sigma^{2}{ }_{\mathrm{d}}=0\right)$, demonstrando adaptação aos ambientes favoráveis e desfavoráveis e previsibilidade de comportamento. São cultivares capazes de responder de maneira satisfatória à melhoria do ambiente e de apresentar alta capacidade produtiva em condições ambientais adversas. Entretanto, DM 247 não deveria ser cultivado nestes ambientes, uma vez que revelou baixo rendimento de grãos.

Os cultivares M-SOY 8866, M-SOY 8914 e FT 106 apresentaram coeficiente de regressão superior à unidade 
Tabela 4 - Médias de rendimento de grãos de cultivares de soja, soja em três ambientes, no Estado do Tocantins, Entressafra 2006

\begin{tabular}{lcccr}
\hline \multirow{2}{*}{ Genótipos } & \multicolumn{3}{c}{ Ambientes $^{1 /}$} & Média \\
\cline { 2 - 4 } & Formoso I & Formoso II & Formoso III & 1.338 \\
DM Nobre & 1.233 & 1.310 & 1.481 & 1.264 \\
A 7002 & 1.201 & 1.113 & 1.447 & 1.110 \\
M-S0Y 8411 & 1.153 & 1.216 & 960 & 1.043 \\
DM 339 & 1.073 & 1.013 & 1.043 & 1.174 \\
DM 309 & 1.050 & 1.200 & 1.271 & 1.256 \\
M-SOY 9350 & 1.070 & 1.260 & 1.440 & 1.132 \\
M-SOY 8866 & 856 & 1.270 & 1.269 & 1.285 \\
M-SOY 8914 & 1.031 & 1.433 & 1.390 & 1.001 \\
FT 106 & 773 & 1.105 & 1.127 & 823 \\
DM 247 & 800 & 790 & 879 & 1.142 \\
Média & 1.023 & 1.171 & 1.234 & \\
\hline
\end{tabular}

${ }^{1 / F o r m o s o ~ I ~-~ 15 / 05 / 2006 ; ~ F o r m o s o ~ I I ~-~ 30 / 05 / 2006 ; ~ F o r m o s o ~ I I I ~-~ 12 / 06 / 2006 ; ~ C V ~(\%) ~=~ 8,34 ~}$

Tabela 5 - Rendimento médio, estimativas dos coeficientes de regressão $\left(\beta_{1}\right)$, dos desvios da regressão $\left(\sigma_{\text {di }}^{2}\right)$ e coeficiente de determinação $\left(\mathrm{R}^{2}\right)$ dos cultivares de soja no Estado do Tocantins, Entressafra 2006

\begin{tabular}{|c|c|c|c|c|}
\hline Genótipos & Média $^{1 /}$ & $\beta_{1}$ & $\sigma_{\mathrm{di}}^{2}$ & $\mathrm{R}^{2}$ \\
\hline DM Nobre & $1.338 \mathrm{a}$ & $1,1092^{\mathrm{ns}}$ & $2573,93^{\text {ns }}$ & 83,71 \\
\hline A 7002 & $1.264 \mathrm{ab}$ & $0,9663^{\mathrm{ns}}$ & $47087,03 *$ & 30,35 \\
\hline M-S0Y 8411 & $1.110 \mathrm{bc}$ & $-0,6756^{+}$ & $21951,25^{*}$ & 29,94 \\
\hline DM 339 & $1.043 \mathrm{c}$ & $-0,1882^{+}$ & $-2055,69^{\text {ns }}$ & 46,05 \\
\hline DM 309 & $1.174 \mathrm{abc}$ & $1,0438^{\mathrm{ns}}$ & $-3006,84^{\mathrm{ns}}$ & 99,92 \\
\hline M-SOY 9350 & $1.256 \mathrm{ab}$ & $1,6721^{\mathrm{ns}}$ & $35,38^{\mathrm{ns}}$ & 95,52 \\
\hline M-SOY 8866 & $1.132 \mathrm{bc}$ & $2,1100^{+}$ & $6631,25^{\mathrm{ns}}$ & 91,51 \\
\hline M-SOY 8914 & $1.285 \mathrm{ab}$ & $1,8841^{+}$ & $11208,43^{*}$ & 85,36 \\
\hline FT 106 & $1.001 \mathrm{c}$ & $1,7812^{+}$ & $1362,06^{\mathrm{ns}}$ & 94,41 \\
\hline DM 247 & $823 \mathrm{~d}$ & $0,2971^{\mathrm{ns}}$ & $-300,45^{\mathrm{ns}}$ & 43,09 \\
\hline Media geral $\left(\mathrm{kg} \mathrm{ha}^{-1}\right)$ & 1.142 & & & \\
\hline
\end{tabular}

${ }^{1 /}$ Médias seguidas por uma mesma letra maiúscula na coluna, não diferem entre si, a $5 \%$ de probabilidade, pelo teste de Duncan; ${ }^{+}=$significativo a $5 \%$ de probabilidade pelo teste $\mathrm{t} ;{ }^{*}=$ significativo a $5 \%$ de probabilidade pelo teste $\mathrm{F} ;{ }^{\text {ns }}=$ não-significativo $(\mathrm{P}>0,05)$.

$\left(\beta_{1 \mathrm{i}}>1\right)$, revelaram adaptação aos ambientes favoráveis. São cultivares cuja utilização deve ser criteriosa, posto que, em ambientes desfavoráveis, ou seja, em regiões envolvendo baixo nível tecnológico e/ou sujeitas às variações edafoclimáticas, podem ter seus rendimentos reduzidos. Segundo Borém e Miranda (2005), esse tipo de genótipo seria o ideal se as condições ambientais pudessem ser controladas para alta performance. Entretanto, apenas M-SOY 8866 e M-SOY 8914 deveriam ser cultivados nestes ambientes, uma vez que produziram acima da média geral. Quanto à estabilidade, M-SOY 8914 apresentou comportamento instável $\left(\sigma_{\mathrm{d}}^{2} \neq 0\right)$.

Os cultivares M-SOY 8411 e DM 339 apresentaram coeficiente de regressão inferior à unidade $\left(\beta_{1 \mathrm{i}}<1\right)$ e baixo rendimento médio, demonstrando estarem pobremente adaptados aos ambientes desfavoráveis. São considerados rústicos, pois mantém seus rendimentos em condições adversas. Entretanto, por não responder satisfatoriamente à melhoria do ambiente, quando se visa a maximização dos rendimentos, não é interessante para sistemas envolvendo o uso de alta tecnologia. Quanto à 
previsibilidade, apenas DM 339 apresentou desvio da regressão não significativo $\left(\sigma_{\mathrm{d}}^{2}=0\right)$.

Analisando os coeficientes de determinação, observa-se que apenas os genótipos A 7002 (30,35\%), M-S0Y 8411 (29,94\%), DM 339 (46,05\%) e DM 247 (43,09\%) apresentaram $\mathrm{R}^{2}$ abaixo de $80 \%$. Raizer e Vencovsky (1999), trabalhando com cana-de-açúcar, relatam que o coeficiente de determinação superior a $80 \%$ representa baixa dispersão dos dados, indicando alta confiabilidade no tipo de resposta ambiental determinado pelas regressões. Segundo Cruz et al. (2006), esse valor deve ser utilizado como referencial para que a regressão explique satisfatoriamente o comportamento de um genótipo em função de um ambiente.

No método do centróide, após a classificação dos ambientes, foram estabelecidos os valores médios dos quatro pontos referenciais (ideótipos), visando representar os genótipos de máxima adaptabilidade geral, máxima adaptabilidade específica a ambientes favoráveis ou desfavoráveis e os genótipos de mínima adaptabilidade, os quais foram acrescidos na análise (TAB. 6).

Em seguida, utilizou-se a análise de componentes principais envolvendo os 10 cultivares iniciais e quatro outros representativos, que na analise gráfica, representam os quatro centróides em torno dos quais foi avaliada a dispersão dos demais. A obtenção dos autovalores, via metodologia dos componentes principais, partindo dos dados originais incluídos os ideótipos, revelaram que dois componentes principais foram suficientes para explicar proporções superiores a 94\% da variação total (TAB. 7). Uma vez constatada a suficiência de dois autovalores na representação da variação total, a avaliação da posição dos genótipos foi realizada através de figura bidimensional (FIG. 1), conforme preconizado por Carvalho et al. (2002).

$\mathrm{Na}$ análise visual da figura de componentes principais, pode-se observar que os cultivares apresentaram distribuição heterogênea para a produtividade de grãos e que existem pontos de maior proximidade a todos os quatro centróides, possibilitando uma recomendação de
Tabela 7 - Estimativa dos autovalores da técnica de componentes principais e fração cumulativa da variância explicada por estes

\begin{tabular}{ccc}
\hline Raiz & Raiz $(\%)$ & \% Acumulada \\
\hline 1,9623 & 65,41 & 65,41 \\
0,8860 & 29,53 & 94,94 \\
0,1516 & 5,06 & 100,0 \\
\hline
\end{tabular}

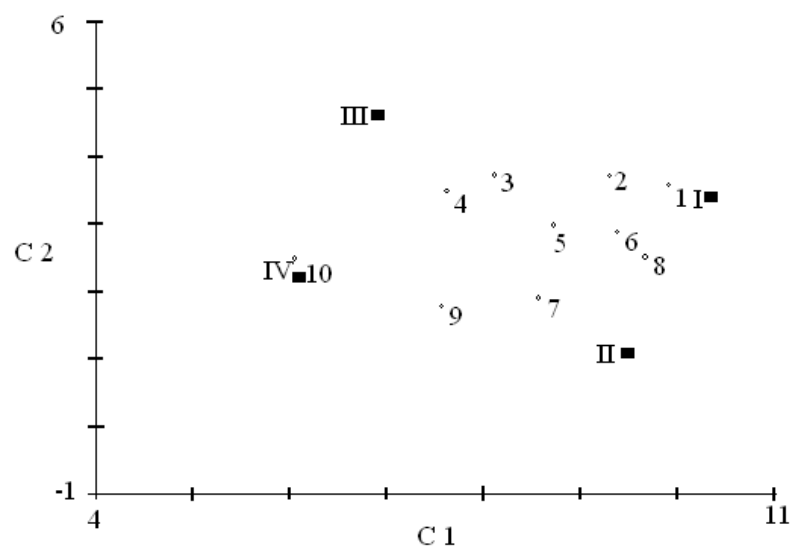

Figura 1 - Dispersão gráfica dos dois primeiros componentes principais da produtividade de 10 cultivares de soja avaliados em três ambientes, Os quatro pontos numerados com algarismos romanos representam os centróides. $\mathbf{1}=\mathrm{DM}$ Nobre; $\mathbf{2}=\mathrm{A}$ 7002; 3 $=$ M-SOY 8411; $\mathbf{4}=$ DM 339; $5=$ DM 309; 6 = M-SOY 9350; $7=$ M-SOY 8866; 8 = M-SOY 8914; 9=FT 106 e 10 = DM 247

Ideótipo I = Adaptabilidade geral (++); Ideótipo II = Adaptabilidade especifica a ambientes favoráveis (+-); Ideótipo III = Adaptabilidade específicaaambientesdesfavoráveis(-+);IdeótipoIV=Poucoadaptado(--)

genótipos de adaptabilidade geral ou recomendação de genótipos de adaptabilidade específica a um subgrupo de ambientes. Os cultivares 1 (DM Nobre) e 2 (A 7002), foram classificados como sendo de adaptabilidade geral, e o cultivar 10 (DM 247) como pouco adaptado, por estarem localizados, respectivamente, mais próximos aos ideótipos

Tabela 6 - Classificação dos ambientes utilizando o índice ambiental e estabelecimento dos ideótipos, calculados pelo método Centróide, dos genótipos de cultivares de soja no Estado do Tocantins, Entressafra 2006

\begin{tabular}{|c|c|c|c|c|c|c|c|c|}
\hline \multirow{2}{*}{ Ambientes } & \multirow{2}{*}{ Média (kg ha'-1) } & \multirow{2}{*}{$\mathrm{I}_{\mathrm{j}}$} & \multicolumn{2}{|c|}{ Produtividade } & \multicolumn{4}{|c|}{ Ideótipos } \\
\hline & & & Máxima & Mínima & $\mathrm{I}$ & II & III & IV \\
\hline Formoso I & 1.023 & $-119,49$ & 1.233 & 773 & 1.233 & 773 & 1233 & 773 \\
\hline Formoso II & 1.171 & 28,31 & 1.433 & 790 & 1.433 & 1.433 & 790 & 790 \\
\hline Formoso III & 1.234 & 91,18 & 1.481 & 879 & 1.481 & 1.481 & 879 & 879 \\
\hline
\end{tabular}

Em que: Ideótipo I = Adaptabilidade geral (++); Ideótipo II = Adaptabilidade especifica a ambientes favoráveis (+-); Ideótipo III = Adaptabilidade específica a ambientes desfavoráveis (-+); Ideótipo IV = Pouco adaptado (--) 
I e IV (FIG. 1). Entretanto, em virtude de a maioria dos pontos (cultivares) estarem plotados na região central da Figura, a classificação via representação gráfica torna-se difícil. Neste caso, foi obtido o inverso do valor da distância entre um ponto aos quatro centróides como estimativa da confiabilidade de agrupamento dos genótipos (ROCHA et al., 2005), ou seja, um ponto eqüidistante aos quatro pontos referenciais apresenta valores de probabilidade de $25 \%$ de pertencer a qualquer um dos grupos e, portanto, quanto mais o valor de probabilidade diferir de $25 \%$, maior será a certeza em concluir o agrupamento do genótipo. Segundo Rocha et al. (2005), valores de probabilidade próximos ou superiores a $50 \%$ indicam boa confiabilidade no agrupamento.

$\mathrm{Na}$ Tabela 8 são apresentadas às classificações dos cultivares a um dos quatro grupos e a probabilidade associada a sua classificação. Os cultivares DM Nobre, A 7002, DM 309, M-SOY 9350 e M-SOY 8914 apresentaram adaptabilidade geral (Grupo I), com destaque para DM Nobre que apresentou probabilidade de 0,643 de pertencer a este grupo; M-SOY 8866 adaptação específica aos ambientes favoráveis (Grupo II); M-SOY 8411 e DM 339 com adaptação específica aos ambientes desfavoráveis (Grupo III) e; FT 106 e DM 247 como de baixa adaptabilidade (Grupo IV).

Observa-se na figura de dispersão (FIG. 1) e na Tabela 8 , uma tendência de aumento na média de produtividade dos cultivares à medida que se aproximam do centróide I (adaptabilidade geral). De acordo com Rocha et al. (2005), quanto menor for a diferença entre um genótipo qualquer e o ideótipo I, menor será a diferença entre este e o genótipo de máximo desempenho em todos os ambientes, fazendo com que a adaptabilidade geral esteja necessariamente associada ao melhor desempenho.

Houve concordância entre as metodologias, quanto à classificação da adaptabilidade dos cultivares, para DM Nobre, A 7002, DM 309 e M-SOY 9350 (adaptabilidade geral); M-SOY 8866 (adaptabilidade específica aos ambientes favoráveis) e; M-SOY 8411 e DM 339 com adaptação específica aos ambientes desfavoráveis.

Os resultados da análise da interação $\mathrm{G} \times \mathrm{A}$ obtidos pelo método do centróide foram comparados com os calculados pelo método proposto por Ebehart e Russel (1966). Considerando que o ideótipo IV representa o grupo dos cultivares pouco adaptados, de nenhum interesse para recomendação e não-contemplado na metodologia de Eberhart e Russell (1966), não foram comparadas as classificações referentes a esse grupo. Os cultivares avaliados por regressão foram ordenados segundo o seguinte critério: I (adaptabilidade geral), $\beta_{1 \mathrm{i}}=1$; II (adaptabilidade específica a ambientes favoráveis), $\beta_{1 \mathrm{i}}>1$; e III (adaptabilidade específica a ambientes desfavoráveis), $\beta_{1 \mathrm{i}}<1$.

Na Tabela 9, foi observado baixo valor da correlação entre os ordenamentos dos genótipos utilizando os valores de probabilidade II do método centróide com os obtidos pelos valores de $\beta_{1 \mathrm{i}}<1$. Entretanto, para os subgrupo I e III houve grande concordância na classificação dos cultivares entre as metodologias. Para o subgrupo III, observou-se correlação intermediaria. Tais valores concordam, em parte, com os obtidos por Rocha et al. (2005), que obteve altas correlações para o primeiro subgrupo e baixas para os demais subgrupos, na cultura do eucalipto (Eucalyptus grandis), e discordam

Tabela 8 - Classificação dos cultivares de soja em um dos quatro grupos caracterizados pelos centróides e a probabilidade associada a sua classificação

\begin{tabular}{lcccccc}
\hline \multicolumn{1}{c}{ Cultivares } & Média & Grupo & Prob (I) & Prob (II) & Prob (III) & Prob (IV) \\
\hline DM NOBRE & 1338 & I & 0,643 & 0,170 & 0,100 & 0,087 \\
A 7002 & 1264 & I & 0,405 & 0,243 & 0,191 & 0,162 \\
M-SOY 8411 & 1110 & III & 0,243 & 0,203 & 0,314 & 0,239 \\
DM 339 & 1043 & III & 0,184 & 0,170 & 0,365 & 0,281 \\
DM 309 & 1174 & I & 0,330 & 0,283 & 0,199 & 0,187 \\
M-SOY 9350 & 1256 & I & 0,436 & 0,296 & 0,137 & 0,130 \\
M-SOY 8866 & 1132 & II & 0,251 & 0,408 & 0,159 & 0,183 \\
M-SOY 8914 & 1285 & I & 0,440 & 0,341 & 0,111 & 0,108 \\
FT-106 & 1001 & IV & 0,196 & 0,268 & 0,214 & 0,322 \\
DM 247 & 823 & IV & 0,244 & 0,270 & 0,056 & 0,892 \\
Media geral (kg ha-1) & 1142 & & & & & \\
\hline
\end{tabular}

Em que: Ideótipo I = Adaptabilidade geral (++); Ideótipo II = Adaptabilidade especifica a ambientes favoráveis (+-); Ideótipo III = Adaptabilidade específica a ambientes desfavoráveis (-+); Ideótipo IV = Pouco adaptado (--) 
Tabela 9 - Estimativa de correlação classificatória de Spearman calculada entre os ordenamentos gerados pelas metodologias centróide e de regressão

\begin{tabular}{lrc}
\hline \multicolumn{2}{c}{ Centróde X Eberhart \& Russell } & R (Spearman) \\
\hline PROB (I) & $\beta_{1 \mathrm{i}}=1$ & 0,80 \\
PROB (II) & $\beta_{1 \mathrm{i}}>1$ & 0,33 \\
PROB (III) & $\beta_{1 \mathrm{i}}<1$ & 1,00 \\
\hline
\end{tabular}

dos resultados obtidos por Peluzio e Sediyama (2000) e Peluzio et al. (2008), que obtiveram alta correlação apenas para o segundo subgrupo, na cultura da soja.

\section{Conclusões}

1. As cultivares DM Nobre, A 7002, M-SOY 9350 e M-SOY 8914 têm bom rendimento de grãos quando cultivados em várzea irrigada;

2. As metodologias de Eberhart e Russell (1966)e Centróide têm, de modo geral, concordância nos resultados, o que proporciona maior confiabilidade na classificação dos cultivares.

3. As cultivares DM Nobre, A 7002, DM 309 e M-SOY 9350 apresentaram adaptabilidade geral; M-SOY 8866 revelou adaptabilidade específica aos ambientes favoráveis e; MSOY 8411 e DM 339 adaptação específica aos ambientes desfavoráveis, em ambas as metodologias.

\section{Referências}

ALLARD, R. W. Relationship between genetic diversity and consistency of performance in different environments. Crop Science, v. 01, n. 02, p. 127-133, 1961.

ALLARD, R. W.; BRADSHAW, A. D. Implications of genotype-environment interactions in applied plant breeding. Crop Science, v. 04, n. 05, p. 503-507, 1964.

BORÉM, A.; MIRANDA, G. V. Melhoramento de plantas. 4. ed. Viçosa: UFV, 2005. 525 p.

CARVALHO, H. W. L. de et al. Adaptabilidade e estabilidade de cultivares de milho no Nordeste brasileiro no triênio de 1998 a 2000. Pesquisa Agropecuária Brasileira, v. 37, n. 11, p. 1581-1588, 2002.

COMPANHIA NACIONAL DE ABASTECIMENTO (CONAB). Cultura da soja no Estado do Tocantins. 2009.
Disponível em: <www.conab.gov.br>. Acesso em: 31 jul. 2009.

CRUZ, C. D. et al. Modelos biométricos aplicados ao melhoramento genético. 4. ed. Viçosa: UFV, 2006. 390 p.

DIAS, F. T. C. et al. Adaptabilidade e estabilidade fenotípica para o caráter rendimento de grãos em cultivares de soja para o Estado do Ceará. Revista Ciência Agronômica, v. 40, n. 01, p.129-134, 2009.

EBERHART, S. A.; RUSSEL, W. A. Stability parameters for comparing varieties. Crop Science, v. 01, n. 01, p. 36-40, 1966.

FINLAY, K. W.; WILKINSON, G. N. The analysis of adaptation in a plant-breeding program. Australian Journal of Agricultural Research, v. 06, p. 742-754, 1963.

PELUZIO, J. M. et al. Análise de regressão e componentes principais para estudo da adaptabilidade e estabilidade em soja. Scientia Agrária, v. 09, n. 04, p. 455-462, 2008.

PELUZIO, J. M. et al. Comportamento de cultivares de soja no Sul do Estado do Tocantins. Bioscience Journal, v. 21, n. 03, p. 113-117, 2005.

PELUZIO, J. M. Interação cultivar $\mathbf{x}$ ambiente e correlações fenotípicas, genotípicas e ambientais entre cultivares de soja no Estado do Tocantins. 1996. 86 f. Tese (Doutorado em Genética e Melhoramento de Plantas) - Universidade Federal de Viçosa, Viçosa.

PELUZIO, J. M.; SEDIYAMA, C. S. Adaptabilidade e estabilidade de produção de grãos de dez cultivares de soja, no estado de Tocantins. Revista Agricultura Tropical, v. 04, n. 01, p. $39-45,2000$.

RAIZER, A. J.; VENCOVSKY, R. Estabilidade fenotípica de novas variedades de cana-de-açúcar para o estado de São Paulo. Pesquisa Agropecuária Brasileira, v. 34, n. 12, p. 2241-2246, 1999.

ROCHA, R. B. et al. Avaliação do método centróide para estudo de adaptabilidade ao ambiente de clones de Eucalyptus grandis. Ciência Florestal, v. 15, n. 03, p. 255-266, 2005.

ROCHA, M. M. et al. Correlações entre parâmetros de adaptabilidade e estabilidade da produtividade de óleo em soja. Ciência Rural, v. 36, n. 03, p. 772-777, 2006.

SILVA, W. C. J.; DUARTE, J. B. Métodos estatísticos para estudo de adaptabilidade e estabilidade fenotipica em soja. Pesquisa Agropecuária Brasileira, v. 41, n. 01, p. 23-30, 2006.

STEEL, R. G. D.; TORRIE, J. H. Principles and procedures of statistics: with reference to the biological sciences. New York: McGraw-Hill Book Company, Inc., 1980. 633 p.

TAI, G. C. C. Genotypic stability analysis and its application to potato regional trials. Crop Science, v. 11, n. 05, p. 84-190, 1971.

VERNETTI, F. de J.; GASTAL, M. F. da C.; ZONTA, E. P. Estabilidade fenotípica de cultivares de soja no sudeste do Rio 